\title{
Operations Specialties Managers
}

National Cancer Institute

\section{Source}

National Cancer Institute. Operations Specialties Managers. NCI Thesaurus. Code C122489.

The group of professions that include administrative services managers, computer and information systems managers, financial managers, human resources managers, training and development managers, and transportation, storage, and distribution managers. 